Review

\title{
Transition from Pediatric Care to Adult Care in Headache Patients
}

\author{
Allyson Bazarsky 1,2, Shalonda K. Slater ${ }^{1,3}$ and Hope L. O'Brien 1,2, \\ 1. Cincinnati Children's Hospital Medical Center, Cincinnati, OH 45229, USA; shalonda.slater@cchmc.org \\ 2. University of Cincinnati College of Medicine, Division of Neurology, Cincinnati, OH 45267, USA \\ 3. University of Cincinnati College of Medicine, Division of Behavioral Medicine and Clinical Psychology, \\ Cincinnati, OH 45267, USA \\ *Correspondence: Hope.OBrien@cchmc.org; Tel.: +1-513-636-4222
}

\begin{abstract}
Migraine is a common condition that affects children as they develop into adults. Transition of care from pediatric to adult care has becoming an increasingly popular topic in the medical literature. It has been suggested that discussions between patients, their families and providers should be initiated as early as age 13. Patients who are un or underprepared have poorer outcomes due to increased morbidity and worsening of their medical condition. Many children continue to have migraine into adulthood and if efforts are taken to ensure patients receive appropriate transfer of care, the results can significantly decrease the economic burden of this disease.
\end{abstract}

Keywords: Transition Medicine; Pediatric migraine; Pediatric; Migraine; Migraine in young adults

Migraine is a common disorder that often begins in childhood. More than $6 \%$ of adolescents are affected in the United States and worldwide as high as $8 \%$ are diagnosed with chronic daily headache $[1,2]$. The diagnosis of headaches in boys occurs between the ages of 10 and 14 years which is earlier than seen in girls who peak at 14-20 years of age [3]. The prevalence of migraine and probable migraine in adults is estimated to be $16.2 \%$ of the US population. The highest burden was seen among women ages 18-44 years. Chronic migraine accounts for over $7 \%$ of the migraine population [4]. Despite the high migraine prevalence, many patients are undiagnosed and/or undertreated due to several factors including lack of access to headache specialty care, delayed or misdiagnosis and poor or suboptimal treatment [3]. Young adults or patients transitioning from pediatric to adult care are most vulnerable to these factors since studies show that most patients who present for an initial visit have had symptoms for on average 10 years [5]. Young adults are less likely to be involved in research studies as the average age involving migraine patients describe pediatric or adolescent (up to the age of 17) or adults where the demographic focus is on women where the average or median age is 40 [6]. Unsuccessful transition can lead to worsening of the disease process and poor outcomes [7].

Transition medicine has been of increasing interest as advances in medicine has led to patients living beyond what has been once considered childhood disease. Emerging research focused on transition of health care in young adults has been evaluated in several different medical subspecialties including congenital heart disease, diabetes, and cystic fibrosis. Early, aggressive and successful treatment of migraine can lead to remission in $18-34 \%$ of adolescents with new onset of migraines [8]. Although remission can be seen in some adolescents, $46 \%$ will have their migraines persist, and $20 \%$ will develop transforming headache [8,9]. These patients will continue to need ongoing headache care and will need to transition care to an adult provider at the appropriate time. However transition care has yet to be explored in the area of headache medicine [10].

There have been several studies attempting to identify patients who would be candidates for successful transition, although this remains a challenge [11-13]. In a study of pediatric cardiac patients, only $47 \%$ had successful transition to the adult cardiac service [12]. The rate of clinical 
attendance in children with type 1 diabetes, which were $94 \%$ for the two years prior to transfer but dramatically dropped to $57 \%$ at 2 years post transfer [11].

Factors attributed to poor transition include lack of preparation or resources targeting late adolescence so many young adults feel unprepared to enter the adult healthcare environment.

Additional factors for migraine patients include having a first degree relative with migraines, delayed diagnosis, difficulty with stress management, poor diet, and obesity [8]. Delayed diagnosis is most often due to unrecognized symptoms or lack of adult features pediatric and adolescent migraines tend to be bi-frontal headaches for a shorter time course [14,15].

Another challenge to the transition process is that in most cases, the process of transitioning patients is not standardized. Each facility is left to decide when or whether a patient is ready to transition and how much guidance he/she will receive during the process. Where there is a lack of guidance, transition is less likely to be successful [16].

Factors attributed to successful transition include patients of an older age at their last visit in pediatric center, those with comorbid conditions, no substance use, and adhered to using prophylaxis antibiotics with dental procedures. Attending appointments without parents or siblings, living close to the medical center, and having well documented recommendations in the patient's chart also correlated with successful transfers. Additionally, success of the transition was not related to age, gender, residence in parent's home, family income, or educational level [12].

A multidisciplinary team of experts developed guidelines on the transition of pediatric to adult healthcare transfers [16]. A consensus statement of eight principles common to a successful transition was created and endorsed by the American Academy of Neurology and Child Neurology Society.

Per their recommendation, discussion of transition should start from a year age, "no later than youths' 13th birthday" which allows time for the patient and caregiver to understand what is required for transition [16] (p. 836). The patient should be provided information on goals such as expanding personal knowledge of their disease process, tools for self-management, and basic understanding of their healthcare system [16,17]. Some understanding over health coverage and insurance is also important since having a change in coverage at time of transition hinders the process and lead to poor outcomes [24]. Overall the goal is to promote skills for self-advocacy. After a discussion has occurred the provider needs to provide documents, such as handouts, which summarize the key points to the patient and caregiver. All written and discussed material needs to be communicated at an age appropriate level for the patient to understand. It is also important the healthcare provider document in the medical record when a discussion of transition occurs [18].

Self-management by the patient is a critical component of transitioning from pediatric to adult healthcare. Beginning at age 12, these skills should be readdressed yearly until the time of the transition. Self-management of a headache disorder includes understanding of the diagnosis, responsibility of self-care, and the ability to make informed decisions [16]. There is no standardized approach to assessing self-management but a checklist might be considered. The patient needs to understand they type of headache disorder they have and how to prevent and abort their headaches. They should also be aware that prevention of headaches consists of healthy habits in the form of life style modification, bio-behavioral approaches including cognitive behavioral therapy and biofeedback, and pharmacologic management [19-21].

Engagement of the patient and family "in phased transition planning, patient education, and transfer readiness" should begin by 13 years of age [16] (p. 836). Patients should be addressed directly and clearly spoken to instead of focusing on addressing the caregivers. Placing the young adult at the center of conversation not only allows the clinician to gain understanding of the patient's knowledge base but also provides direct evidence for the patient that more responsibility is expected of them. The yearly assessment of the level of engagement should include but not be limited to their medical condition, medications and understanding side effects, identifying significant signs and symptoms, and assess their psychological health [16]. The Transition Readiness Assessment Questionnaire (TRAQ) is a validated tool that is widely used to help providers identify areas to address where a patient may not feel ready to transition [7,10]. The TRAQ addresses five categories: 1) managing medications, 2) appointment keeping, 3) tracking health issues, 4) talking with providers, and 5) 
managing daily actives [7]. It is considered the "best validated transition-readiness tool" when compared with ten assessment tools for adolescents with chronic disease [22] (p. 1). The TRAQ is preferred because it is disease neutral, has two main domains of skills of self-management and skills for self-advocacy, has internal consistency, and has undergone more testing than the majority of transition readiness tools $[22,23]$. The patient should fill out the questionnaire independently to order to track their own abilities against themselves overtime. This will provide insight on areas that need improvement in order for the young adult to make a successful transition in the future.

When the child reaches the age of 14 a transition plan should be developed with the collaboration of the patient, caregivers, and involved healthcare professional, and any other involved parties. The provider may want to consider a "transfer packet" customized for the headache patient consisting of information about the headache history, diagnoses, treatment plan including failed medication, length of medication trials, an emergency plan, comorbid disorders like anxiety or depression, and non-pharmacological headache treatments utilized. The patient's emergency plan can be written in the form of an emergency department letter, which the individual can supply, to a provider if he/she needs to seek out emergent care. A list of preferred medications should be summarized with specifics on dosing and frequency [16] (p. 837). It would be also advantageous to list medications which should be avoided that can lead to rebound or worsen headache disorders. The packet can then be used for coordination of care and communication between providers [16].

The transition plan of care should be refined and verified on an annual basis and should also include the patient's goals and preferences. During these yearly discussions the timing of transition should be considered [16].

Prior to transition, the pediatric provider, the patient and if relevant, the caregiver(s) should work together to identify an adult provider who would be appropriate to take over managing the patient's headaches [16]. The ability to ascertain an adult provider can be very challenging for young adults. Major obstacles include understanding how health insurance is involved with choosing a provider, choosing a provider who is a headache specialist versus a general neurologist, not to mention the anxiety related to speaking with an unfamiliar provider. If an adult provider can be identified 1-2 years prior to transition it can reduce the associated stress and anxiety and in such case, the young adult might consider a preliminary visit with an adult provider if needed to allow for a successful transition [24,25].

When the young adult is ready for transition the pediatric provider should have direct communicate with the adult provider and offer to provide a transfer packet regarding the patient's care. An appointment should be scheduled and attended by the young adult, but the pediatric provider is responsible for confirming a successful transition has been made and adult care is establish [16]. To make this successful patients should feel comfortable making phone calls to providers on their own behalf for scheduling and to discuss their condition.

For young adults going to college, it is important to identify where they want their care to occur. Many college students request to have their physicians and healthcare at home where their families live instead of where the school is located. For those who plan on moving or attend college at a distance, identifying a specialist in the area they plan to reside early in the process could help avoid disruption in headache management. Patients may also request follow-up visits with an alternate adult provider should they return home during school breaks.

In summary, successful transition of pediatric headache care to an adult provider can be challenging and often time consuming, for both patients and providers, but is vitally important. Patients receiving a diagnosis and treatment at young age and will continue to need to management of their headaches throughout their adult life. Providing a patient with the confidence and skills starting a young age, around 13 years of age, will set the young adult for successful transition from pediatric to adult headache care. Furthermore, avoiding breakdowns in the transition process can decrease longer term morbidity and potentially reduce the chronification of many headache disorders.

Acknowledgments: No funding was received towards production of this manuscript. 
Conflicts of Interest: Dr. Allyson Bazarsky is a headache medicine fellow who has no conflict of interest to disclose. Dr. Shalonda Slater is an associate professor who has not conflict of interest to disclose. Dr. Hope O'Brien is an associate professor has received honorarium for published work for Up to Date and Turner White communications. She is also on the speaker bureau for Avanir.

\section{References}

1. Bigal, M.E.; Lipton, R.B.; et al. Migraine in adolescents - association with socioeconomic status and family history. Neurology 2007, 69, 16-25.

2. Chong, S.C., Chan, Y.H.; et al. Headache diagnosis, disability and co-morbidities in a multiethnic. Heterogeneous peadiatric Asian population. Cephalalgia 2010, 30, 953-961.

3. Hershey A.; Powers, S.; et al. Pediatric Headaches in Clinical Practice, $1^{\text {st }}$ ed.; John Wiley \& Sons: Oxford, UK, 2009; pp 1-99.

4. Simtherman, T.A.; Burch, R.; et al. The Prevalence, Impact, and Treatment of Migraine and Severe Headaches in the United States: A Review of Statistics From National Surveillance Studies. Headache 2013, 53, 427-436.

5. Murtaza, M.; Kisat, M.; et al. Classification and Clinical Features of Headache Disorders in Pakistan: A Retrospective Review of Clinical Data. PLoS ONE 2009, 4(6), e5827. doi:10.1371/journal.pone.0005827.

6. Termine, C.; Özge, A.; et al. Overview of diagnosis and management of paediatric headache. Part II: therapeutic management. J Headache Pain 2011, 12, 25-34.

7. Wood A.L.; Sawicki, G.S.; et al. The Transition Readiness Assessment Questionnaire (TRAQ): Its Factor Structure, Reliability, and Validity. Academic Pediatrics 2014, 14, 415-422.

8. Monastero, R.; Camarda, C.; et al. Prognosis of migraine headaches in adolescents: A 10-year follow up study. Neurology 2006, 21, 327-332.

9. Congdon, P.J.; Forsythe, W.I. Migraine in childhood: A study of 300 children. Dev med child Neurol 1979, 21, 209-216.

10. Sawicki, G.S.; Lukens-Bull, K.; et al. Measuring the Transition Readiness of Youth with Special Healthcare Needs: Validation of the TRAQ-Transition Readiness Assessment Questionnaire. Journal of Pediatric Psychology 2011, 36(2), 160-171.

11. Kipps, S.; Bahu, T.; et al. Current methods of transfer of young people with Type 1 diabetes to adult services. Diabetic Medicine 2002, 19, 649-654.

12. Reid, G.J.; Irvine, M.J.; et al. Prevalence and Correlates of Successful Transfer From Pediatric to Adult Health Care Among a Cohort of Young Adults With Complex Congenital Heart Defects. Pediatrics 2004, 113(3), e197-205.

13. O'Brien, H.L.; Cohen, J.M. Young Adults With Headaches: The Transition From Adolescents to Adults. Headache. 2015, 55, 1404-1409.

14. Virtanen, R.; Aromaa, M.; et al. Changing headache from preschool age to puberty. A controlled study. Cephalagia 2007, 27, 294-303.

15. Bigal, M.E.; Arruda, M.A. Migraine in the Pediatric Population-Evolving Concepts. Headache 2010, 50, 11301143.

16. Brown, L.W.; Camfield, A.; et al. The neurologist's role in supporting transition to adult heath care: a consensus statement. Neurology 2016, 87, 835-840.

17. Kennedy, A.; Sloman, F.; et al. Young people with chronic illness: the approach to transition. Internal Medicine Journal 2007, 37, 555-560.

18. Cooley, W.C.; Sagerman, P.J. Supporting the health care transition from adolescence to adulthood in the medical home. Pediatrics 2011, 128, 182-200.

19. Hershey A.D. Current approaches to the diagnosis and management of paediatric migraine. Lancet Neurology 2010, 9, 190-204.

20. Powers, S.W.; Andrasik, F. Biobehavioral treatment, disability, and psychology effects of pediatric headache. Pediatr Ann 2005, 34, 461-464.

21. Powers, S.W.; Kashikar-Zuck, A. M.; et al. Cognitive behavioral therapy plus amitriptyline for chronic migraine in children and adolescents: A randomized clinical trial. JAMA 2013, 33(8), 16-17.

22. Zhang, L.F.; Ho J.S.W.; Kennedy, S.E. A systematic review of the psychometric properties of transition readiness assessment tools in adolescents with chronic disease. BMC Pediatrics 2014, 14(4), 1-10. 
23. Schwartz, L.A.; Daniel, L.C.; et al. Measures of Readiness to Transition to Adult Health Care for Youth with Chronic Physical Health Conditions: A systematic Review and Recommendations for Measurement Testing and Development. Journal of Pediatric Psychology 2014, 39(6), 588-601.

24. Rutishauser, C.; Sawyer, S.M.; Ambresin, A.E. Transition of young people with chronic conditions: a crosssectional study of patient perceptions before and after transfer from pediatric to adult health care. Eur J Pediatr 2014, 173, 1067-1074.

25. Oskoui, M.; Wolfson, C. Treatment Comfort of Adult Neurologists in Childhood Onset Conditions. Can J Neurol Sco 2012, 39, 202-205. 